\title{
A More Reliable Step Counter using Built-in Accelerometer in Smartphone
}

Win Win Myo, Wiphada Wettayaprasit, Pattara Aiyarak

Department of Computer Science, Faculty of Science, Prince of Songkla University, Hat Yai, 90110, Songkhla, Thailand

\begin{tabular}{l} 
Article Info \\
\hline Article history: \\
Received Apr 9, 2018 \\
Revised May 20, 2018 \\
Accepted Jul 11, 2018 \\
\hline
\end{tabular}

Keywords:

Step counter

Accelerometer

Sensor

Smartphone

Mobile phone

\begin{abstract}
Step counter, being an active area of human daily physical activity, is an essential role in human activity determination research. As the current smartphones come with many different sensors and powerful processing capabilities, the step counting using built-in sensors in a smartphone is increasingly becoming a vital factor among many researchers. However, the step counting with a smartphone has still challenging due to many different walking behaviors and mobile phone positions. In this study, we introduce a more reliable step counter's technique using Accelerometer sensor in a smart phone. The objective of this study is to get the accurate steps of three different walking activities in four different mobile positions. In order to achieve this, a new reliable technique based on peak is attracting considerable in our work using average acceleration. The experimental result shows $99.02 \%$ as an overall step counting performance that the proposed method reliably detects the steps under varying walking speed in different devices modes. This result is encouraging to facilitate among of the complex walking activities using built-in sensors in smartphone.
\end{abstract}

Copyright $\odot 2018$ Institute of Advanced Engineering and Science. All rights reserved.

\section{Corresponding Author:}

Pattara Aiyarak,

Department of Computer Science, Faculty of Science,

Prince of Songkla University,

Hat Yai, 90110, Songkhla, Thailand.

Email: pattara.a@psu.ac.th

\section{INTRODUCTION}

Step counter is an automatic detection of walking steps taken by a human. It is a part of human activity recognition or better walking detection. Step counting is becoming a very important role in healthcare system to monitor the human's daily routine. In the current age of technology, step counting technique using built-in sensors in a smartphone has been becoming progressive and important area due to coming with many embedded sensors such as accelerometer, gyroscope, magnetometer which provide valuable information of user's contexts and activities.

Many researchers have been approached the step counting techniques by built-in a variety of sensors in mobile phones with different points of views. However, there are many challenges to motivate a new step counter technique under more realistic conditions. The following are some of the step counter's challenges.

1) Human behavior: Performing multiple tasks during walking at the same time [1]-[3]

2) Sensor data: Being very noisy data [3]

3) Sensor orientation: Changing the sensor position during motion [2], [3]

4) Usability: Necessity for easier system to be more efficient[3]

5) Privacy: Necessity reliability of user private information [3]

6) Subject sensitivity: Depending on participation of subjects in training and testing stages to get the accurate results[2] 
7) Data collection: Necessity of the realistic condition for collecting data[4], [5]

These challenges support our decision to do this work. Our motivation is to find an accurate step counting technique without considering in different walking speeds and mobile positions. We propose a more reliable step detection technique for human walking activities by using a built-in Accelerometer sensor in a smartphone. This proposed model has to intend to detect the steps under varying walking modes and different mobile positions. The ideas behind this approach are:

1) To judge easily the steps of a human

2) To handle the different manners of a human

3) To place the smartphone in many different locations

4) To support in health care system

The paper is structured with five sections. The section two gives a brief overview of some background information and related works. A new methodology is outlined in the section three. The section four looks at the experimental results and investigates the solutions. The conclusions, outcomes, and direction for future work are drawn in the section five.

\section{BACKGROUND INFORMATION AND RELATED WORKS}

The step counting is a critical part of doing regular physical activity. A sensor is a device, which can detect and respond to some type of input from the physical environment such as light, sound, heat, moisture, motion, pressure, and so on. The output of sensor is generally a signal, which can convert to human-readable data for reading or further processing. Although the earlier smartphone has a single sensor (Accelerometer), the current mobile phones come with built-in multi-sensors such as accelerometer, gyroscope, magnetometer, Wi-Fi, Bluetooth, etc. These sensors can measure a physical quantity such as motions, orientations, and various environmental conditions and provide some valuable information to the user. There are three kinds of sensors: motion sensors, environmental sensors, and position sensors. Accelerometers, Gyroscopes, Gravity sensor, and Rotational Vector sensor are motion sensors. Barometers, Photometers, Thermometers, Wi-Fi, and Bluetooth sensor are environmental sensors, which can measure various environmental parameters. The orientation sensors and magnetometers are positioned sensors, which can measure the physical position.

As the smartphone is provided with the easy programming interface, large storage, and processing capabilities, the human activity determination using built-in sensors in a smartphone is becoming a rapidly growing field. In [6], the authors discussed about the human activity recognition on a smartphone. On the other hand, the mobile application can achieve information for desired tasks. The mobile sensing has increased significantly and archived the benefits of human society. The authors in [7] mentioned the benefit of mobile sensing and introduced the trash basket sensor to protect the environment from the pollution negative effects. Because of their ubiquitous nature signal, the mobile phone is used as an essential and an ideal medium for recognizing human physical activities and many other research areas. Therefore, the researchers from many fields have been attracting attention in sensing mobile phones.

As well as step counting is very important to monitor human health, it can provide many applications such as many medical fields, fitness tracking center, human activity recognition system and so on. In previous, more reliable and electronic devices called pedometers are utilized, especially in medical applications. In recent years, pedometers are used as the electronic step counting devices, which are attached to waist, foot, or arms. Currently, the step counting has become an increasing research area with the powerful capacities of smartphones sensors, including gyroscope and accelerometer. Typically, the accelerometer and gyroscope sensors are highly effective for step counting. Both sensors are suitable for motion analysis, but accelerometer is more popular as its ability detects the rate of change in motion activity. In fact, sensor location and orientation are very important factors to be a more effective step counting in human daily activity. The authors in [8] introduced a novel step counting algorithm based on acceleration and gravity sensors. They regardless the orientation of smart phone and user motion. They showed that their performance is more suitable than the four different applications.

Step counting is important for a person's rehabilitation improvement. In [9], the authors have studied the gait and activity recognition. They distinguished about specific walking speed (slow, normal, and fast) by placing a mobile phone in their trousers' pockets and they achieved the accuracy $93.6 \%$ in slow walking, $71.2 \%$ in normal walking and, $94.4 \%$ in fast walking. They showed the highest performance in the fast walking activity. The authors in [10] proposed a new step counting algorithm based on the Discrete Kalman filter to detect the steps via iPhone's accelerometer carrying in T-shirt pocket and on hand mode. They proved the average accuracy of $96.3 \%$ in on hand mode and $96.7 \%$ in pocket mode. The authors in [11] suggested a step counting method using Fast Fourier Transform and threshing for 3 activities (sitting, standing, and walking) using Android smartphone accelerometer. Their results showed the average performance accuracy of $87.52 \%$ in walking activity and $41.7 \%$ in running activity. 
The Pedometer applications on a smartphone are used by either Accelerometer or Gyroscope. The authors in [12] considered the daily step counting recorded by Accelerometer and compared with Pedometer and achieved averaged accuracy over $60 \%$ per day. In [13], the authors proposed a new robust and accurate step counting technique by overcoming false walking and they considered for 7 positions (texting, calling, pocket, swinging, handbag, backpack, arm-band) with 3 activities (walking, running, free walking). Their result showed the average accuracy of $98.7 \%$ for any walking modes and any positions. The authors in [14] introduced a novel pedometer to get a better accuracy in step counting using in different mobile position (texting, shirt pocket, pant pocket. Hand swinging). They got the overall accuracy 98.73\%. As the measurement of physical activity, especially for older patients, is needed to improve their quality of life, the authors in [15] investigated the accuracy of pedometer using Accelerometer for slow walking speeds. The authors in [16] concentrated a novel technique to find the total distance walked, step timing, gait symmetry using Accelerometer and Gyroscope during a 6-minute walking test for chronic obstructive pulmonary disease and congestive heart. Although many studies have proven for counting steps, the monitoring is not accurate yet. The authors in [17] attempted to monitor the natural walking for pulmonary patients using Accelerometer. All attempts of their works have to consider different walking speeds and varying usage modes. Therefore, the proposed method considers being a reliable step counting in every walking speed crossing the every usage mode.

\section{METHODOLOGY}

The system schematic diagram is shown in Figure 1, which supports our prior proposed method to get accurate steps. We emphasized that our proposed method will effectively point out the step points in any user's motion styles and mobile phone's positions.

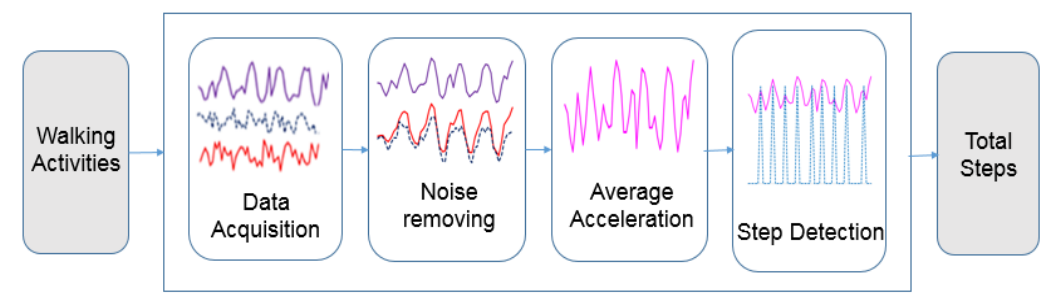

Figure 1. System architecture of the proposed model

Figure 1 demonstrates with three processes how our system will develop. It starts with a data acquisition using a built-in Accelerometer in Samsung Galaxy Note 4 with Android 6.0.1 operating system. The data collection involves three different walking styles (normal, slow, and fast) in four different usage modes (swinging mode, texting mode, waist-bag mode, and arm-bag mode). Therefore, there are twelve categorizations in our experiment for data collection as described in Table 1. Based on these guidelines, we have selected nine subjects (6 males, 3 females) to perform in our experiment as mentioned above. Each person has to walk 30 steps for every activity and the sensor retrieves the data in every $100 \mathrm{~ms}$. All users' activities are captured by video.

Table 1. The twelve walking activities for data acquisition

\begin{tabular}{|c|c|c|c|}
\hline $\begin{array}{c}\text { Smartphone } \\
\text { Positions }\end{array}$ & \multicolumn{3}{|c|}{ Walking Styles } \\
\hline Swinging mode & Normal & Slow & Fast \\
\hline Texting mode & Normal & Slow & Fast \\
\hline Waist-bag mode & Normal & Slow & Fast \\
\hline Arm-bag mode & Normal & Slow & Fast \\
\hline
\end{tabular}

The second stage is the data preprocessing. In [18], they used low-pass filter to split the acceleration data to low frequency and high frequency. In our work, we use low pass filter to smooth the sensor signals. Our approach considers on average acceleration value described in equation (1) which has a sinusoidal 
pattern. In fact, the frequency data of the signal tell historically what proportion of every frequency exists in time. Besides, the information of the signal does not change within the signals, and thus it is affected by the filtering operations [19].

$$
\|a\|=\sqrt{x^{2}+y^{2}+z^{2}}
$$

Where $a$ is average acceleration value; $x, y$, and $z$ are three axis values.

The final stage is a key to complete our proposed model, providing the accurate steps. We use a new reliable technique based method of peak. In frequency data of the average acceleration signals, the method finds the peaks points, which is greater than the other points. Then the method checks the number of the occurrence point between two peaks. In which, the number of occurrence points must be greater than two. If the peak point satisfies this condition, it is identified as a one-step. All step points are picked according to the principle rule of the proposed method. The step counting is subsequently taken over one-step to another until reaching the stopping criterion. The complete algorithm of the proposed method to detect the steps describes in the following Figure 2.

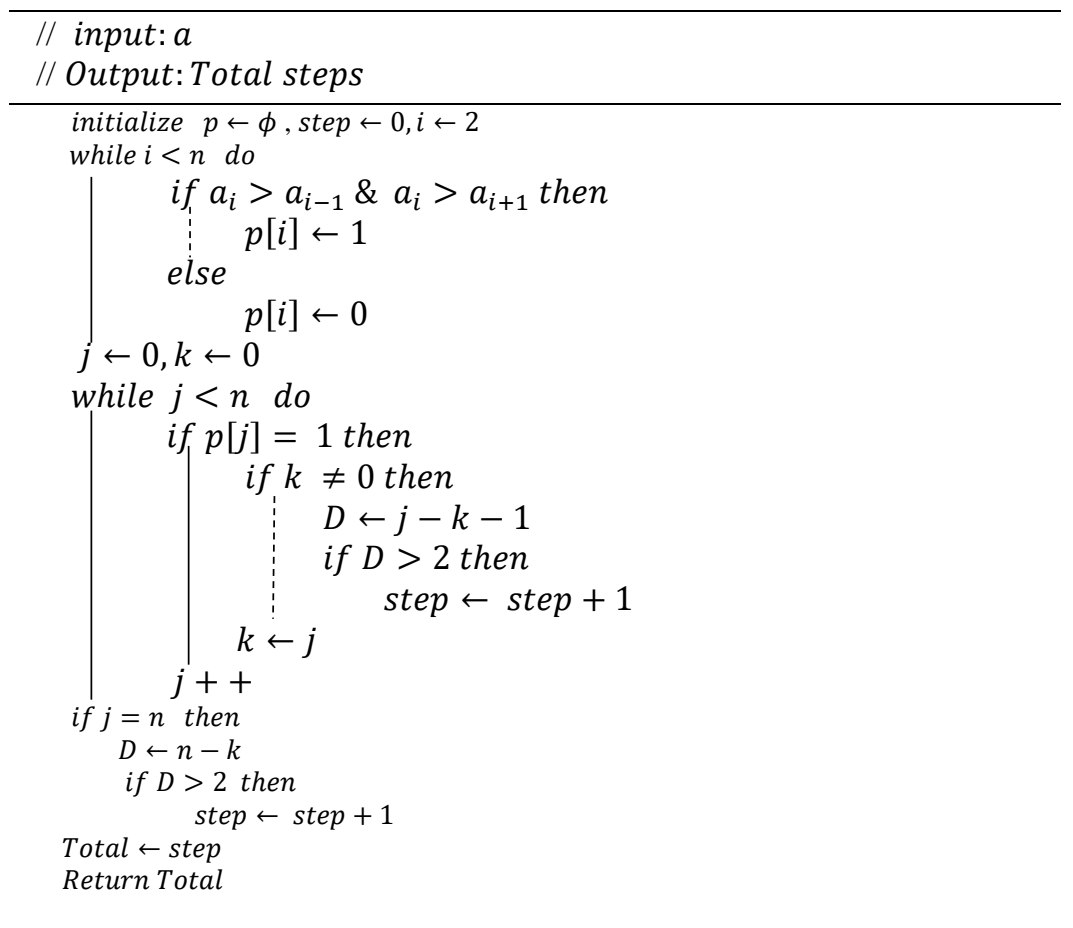

Figure 2. The step detection algorithm

Figure 2 illustrates the algorithm how to find the total steps. The input parameter ' $a$ ' is raw signal data of accelerometer sensor. The parameter $n$ is the total number of raw data. The parameter $p$ is the local maxima point. The parameter $\mathrm{D}$ is the number of occurrence points during two points. The peak point is developed and compared by the before and after of this point. Then the number of occurrence points is calculated. If it is greater than 2 , the step will be counted and returned to the 'Total'.

\section{RESULTS AND DISCUSSIONS}

There are several factors to search out the accuracy of steps in different walking activities (normal, slow, fast) and usage modes (swinging mode, texting mode, waist-bag mode, arm-bag mode) of mobile phone as shown in Figure 3, Figure 4, and Figure 5. All factors include the different raw signals retrieved by Accelerometer sensor from a Samsung Galaxy Note 4 and step signals detected by the proposed algorithm.

Figure 3 shows the sample raw signals of three different walking activities and the step detections in hand-swinging mode as explained above. In Figure 3(a), 3(b), and 3(c), the red line, the purple line, and the 
dotted blue line represent the raw signal data of Accelerometer. These signals come individually and are different each other on which the $\mathrm{x}$ value is the biggest one and $\mathrm{y}$ value is the smallest one. Then the pink line represents the average acceleration value and the dotted blue lines represent the detected steps of walking activity. This illustration indicates that the walking steps are thoroughly checked by using the proposed method. Figure 4 demonstrates the sample raw signals of three different walking activities and the step detections in texting mode. In Figure 4(a), 4(b), and 4(c), the red line, purple line and, the dotted blue line represent the raw data of Accelerometer. It shows that the $\mathrm{z}$ value is significantly the biggest one. Then the pink line represents the average acceleration value and the dotted blue lines represent the identified steps of user's walking. In which, the steps are gently detected due to a reliable technique. Figure 5 defines the raw signals of three different walking activities and the step detections of these walking in waist-bag mode. In Figure 5(a), 5(b), and 5(c), the red line, purple line, and the dotted blue line represent the raw signal of Accelerometer. In which, we can see that the $\mathrm{z}$ value leads to $\mathrm{x}, \mathrm{y}$ values and $\mathrm{x}$ value leads to $\mathrm{y}$ value. Then, the pink line represents the average acceleration value and the dotted blue lines represent the detected steps of user's walking. Here, we can see that the proposed technique definitely traces in different walking steps.

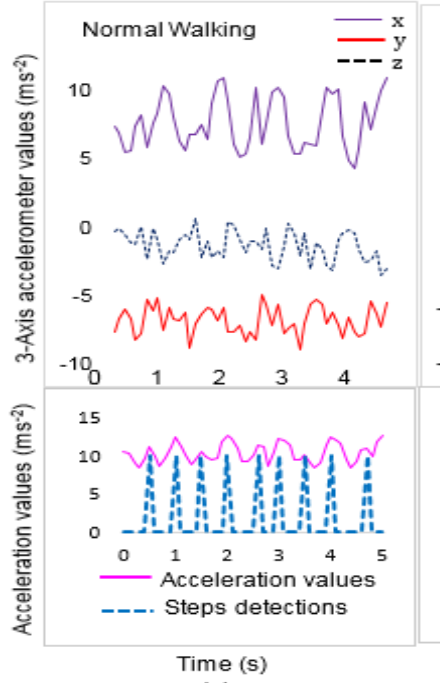

(a)

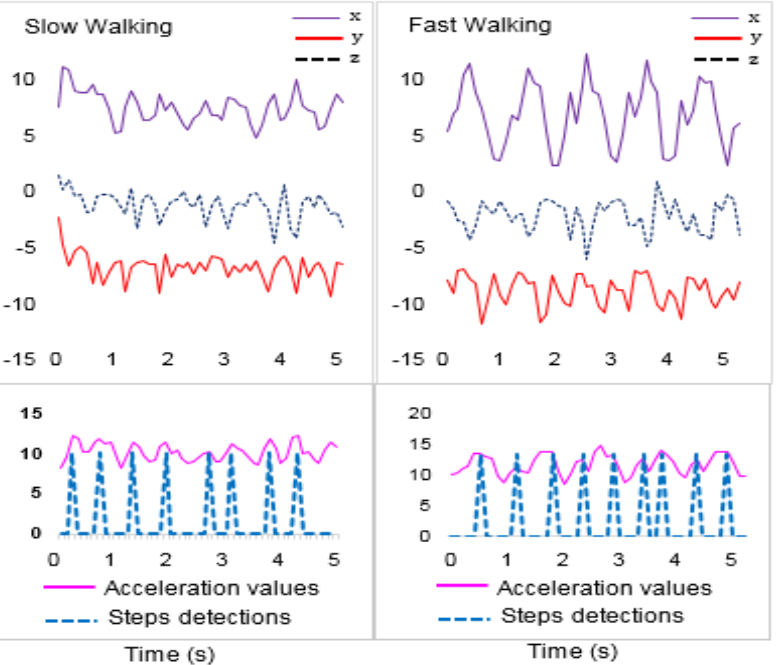

(c)

Figure 3. Raw signals and step signals of three different walking activities in swinging mode

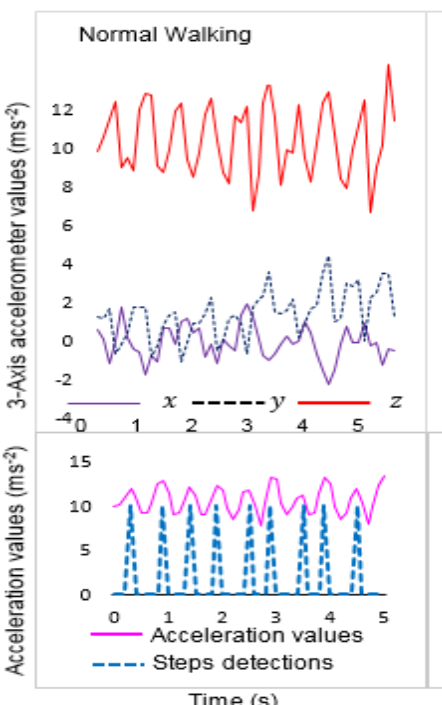

(a)
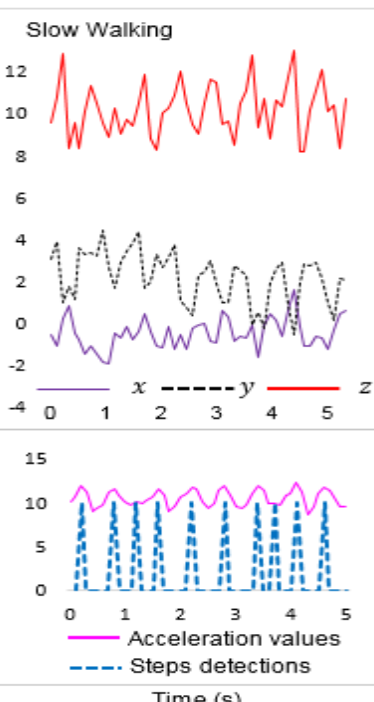

(b)

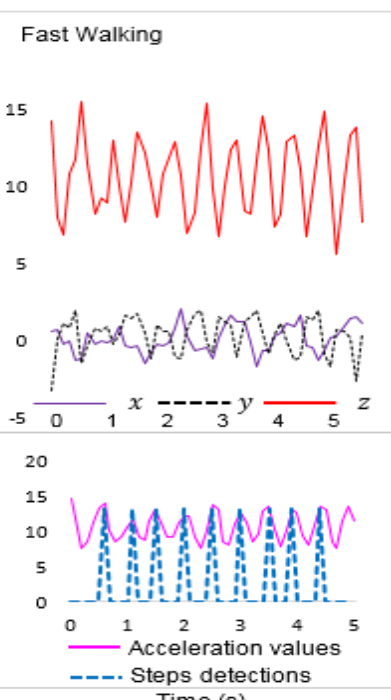

ime (s)

Figure 4. Raw signals and step signals of three different walking activities in texting mode 


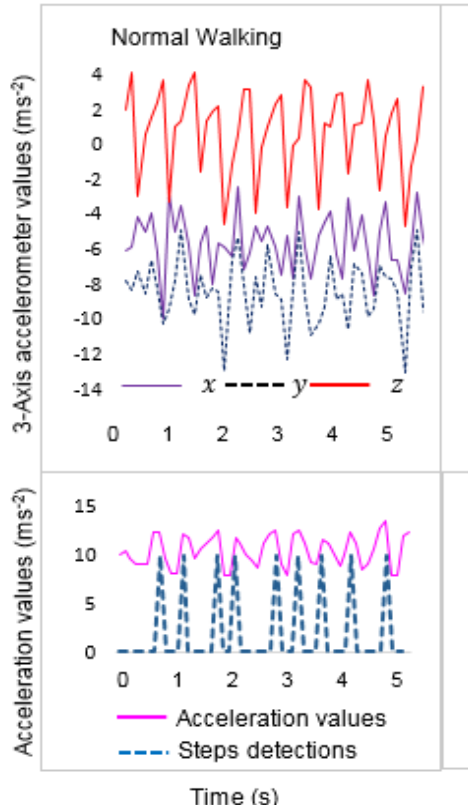

(a)

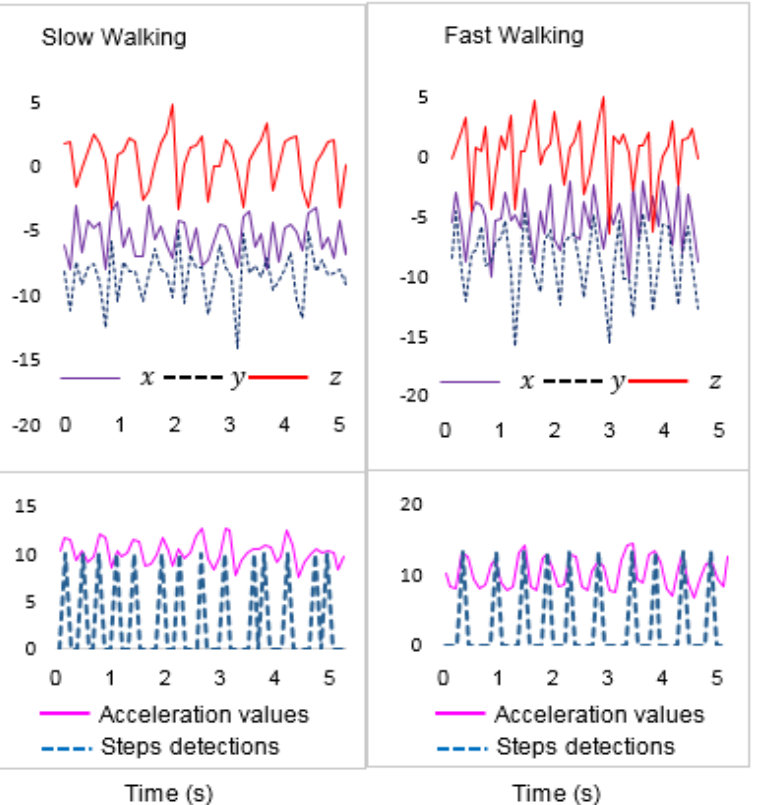

(b)

(c)

Figure 5. Raw signals and step signals of three different walking activities in waist-bag mode

Then we can conclude that the raw signals of three axes vales come individually in hand swinging mode because the accelerometer sensor has to measure not only the movement acceleration due to walking of user's steps but also the component acceleration due to the hand swinging motion. On the other hand, the two raw signal data are closely come together in static positions of smart phone such as texting mode and waistbag mode. During the experiment, the number of steps, as counted by the proposed method, is saved for every activity. This study also performs the elaboration of recorded video of every user's walking. As a final stage, the calculated step counts of the proposed designed scheme are compared with the elaborated step counts of video (real step counts of each user). This supports the proposed model to be a more reliable step counter. The comparative analysis result of nine users is described in Table 2.

Table 2. Comparative results of the actual steps (A) and the predicted steps (P)

\begin{tabular}{|c|c|c|c|c|c|c|c|c|c|c|c|c|c|c|c|c|c|c|c|c|c|c|c|c|}
\hline \multirow{3}{*}{ User } & \multicolumn{6}{|c|}{ Swing mode } & \multicolumn{6}{|c|}{ Texting mode } & \multicolumn{6}{|c|}{ Waist-bag mode } & \multicolumn{6}{|c|}{ Arm-bag mode } \\
\hline & \multicolumn{2}{|c|}{ Normal } & \multicolumn{2}{|c|}{ Slow } & \multicolumn{2}{|c|}{ Fast } & \multicolumn{2}{|c|}{ Normal } & \multicolumn{2}{|c|}{ Slow } & \multicolumn{2}{|c|}{ Fast } & \multicolumn{2}{|c|}{ Normal } & \multicolumn{2}{|c|}{ Slow } & \multicolumn{2}{|c|}{ Fast } & \multicolumn{2}{|c|}{ Normal } & \multicolumn{2}{|c|}{ Slow } & \multicolumn{2}{|c|}{ Fast } \\
\hline & $\mathrm{A}$ & $\mathrm{P}$ & A & $P$ & $\mathrm{~A}$ & $P$ & $\mathrm{~A}$ & $P$ & A & $\mathrm{P}$ & $\mathrm{A}$ & $P$ & $\mathrm{~A}$ & $P$ & $\mathrm{~A}$ & $P$ & $\mathrm{~A}$ & $P$ & $\mathrm{~A}$ & $P$ & $\mathrm{~A}$ & $P$ & $\mathrm{~A}$ & $P$ \\
\hline 1 & 30 & 30 & 31 & 30 & 30 & 30 & 30 & 30 & 31 & 30 & 30 & 30 & 30 & 30 & 30 & 30 & 31 & 29 & 30 & 29 & 30 & 29 & 30 & 30 \\
\hline 2 & 30 & 30 & 31 & 31 & 30 & 29 & 31 & 31 & 31 & 30 & 30 & 29 & 31 & 31 & 31 & 30 & 30 & 30 & 31 & 31 & 31 & 31 & 30 & 30 \\
\hline 3 & 31 & 31 & 31 & 31 & 30 & 30 & 30 & 30 & 30 & 30 & 29 & 29 & 31 & 30 & 31 & 31 & 30 & 30 & 30 & 30 & 30 & 30 & 30 & 29 \\
\hline 4 & 30 & 30 & 30 & 30 & 30 & 30 & 30 & 29 & 31 & 31 & 30 & 30 & 31 & 31 & 29 & 29 & 30 & 30 & 30 & 30 & 30 & 29 & 30 & 29 \\
\hline 5 & 30 & 30 & 31 & 30 & 30 & 30 & 31 & 31 & 31 & 30 & 31 & 31 & 31 & 31 & 31 & 31 & 31 & 30 & 31 & 31 & 31 & 31 & 31 & 30 \\
\hline 6 & 30 & 30 & 30 & 30 & 30 & 30 & 30 & 30 & 30 & 30 & 30 & 30 & 30 & 30 & 30 & 29 & 30 & 30 & 30 & 30 & 30 & 29 & 30 & 30 \\
\hline 7 & 30 & 29 & 30 & 29 & 31 & 30 & 30 & 29 & 29 & 29 & 30 & 29 & 31 & 31 & 30 & 30 & 30 & 30 & 30 & 29 & 31 & 31 & 30 & 30 \\
\hline 8 & 30 & 30 & 30 & 29 & 29 & 29 & 30 & 30 & 30 & 30 & 30 & 30 & 31 & 31 & 31 & 31 & 31 & 31 & 30 & 30 & 30 & 30 & 31 & 31 \\
\hline 9 & 30 & 30 & 30 & 30 & 31 & 30 & 30 & 30 & 30 & 29 & 30 & 30 & 30 & 30 & 30 & 29 & 31 & 31 & 29 & 29 & 30 & 29 & 30 & 30 \\
\hline
\end{tabular}

On comparing these results, we conclude that the proposed scheme can exactly detect most of users' steps in every activity. This allows a formal solution that all user pace cannot be into activeness level because everyone has its own walking pace relying on their weight and height. However, our proposed method carefully checks for all user' walking steps. Finally, these results cover our target. A significant observation result of our experiment is shown in Figure 6 and Figure 7. 


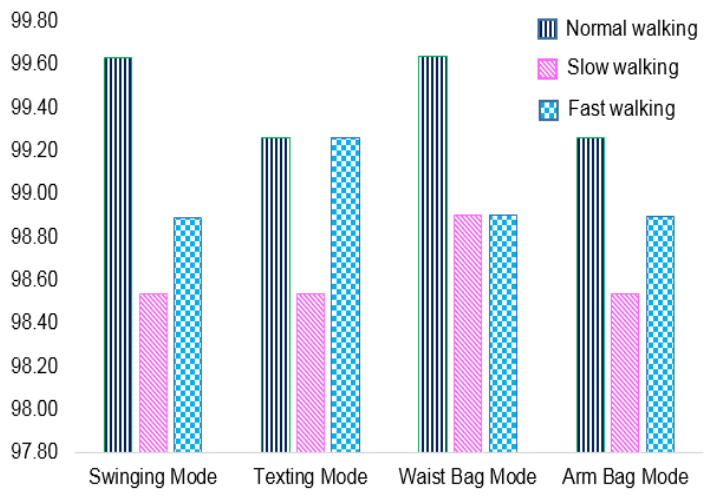

Figure 6. Overall results of three different walking

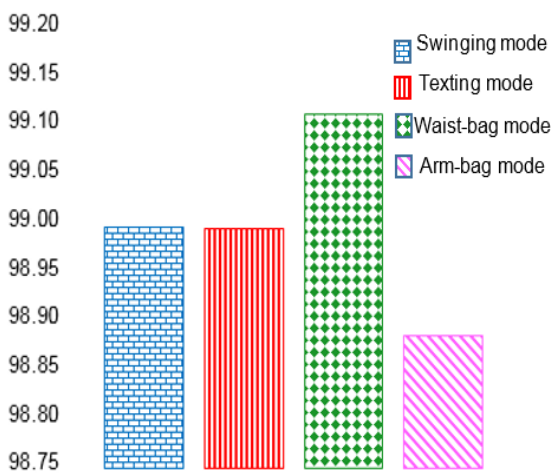

Figure 7. Overall results in four different usage mode

Figure 6 outlines the overall results on three different walkings in four usage modes. It can be seen that the accuracy of normal walking represents the best one, whereas the accuracy of the slow walking activity represents the lower accuracy of others. In Figure 7, the design of the mobile positions is geared towards a better accuracy. By having the stability of smartphone positions, the waist-bag mode accuracy a better one. There has almost same performance in swinging mode and texting mode positions, whereas the performance of the arm-bag mode is rather than others. It is very likely that our proposed method is able to investigate a major source of reliability of the step counting due to the average performance result of $99.02 \%$. We highlight that our research is unable to consider for two limitations (user's motion and mobile phone's position).

\section{CONCLUSIONS}

The proposed model has presented as a more reliable step counter technique using built-in accelerometer sensor in the smartphone. The proposed system considers typical methods on the average acceleration values with low pass filter. By using rule based method of peak detection, the outcomes shows that our proposed method is able to find a reliable step counter for human daily activity. The evidence from this study suggests that our approach is capable of performing in every step counting of human daily life. To sum up, we can identify the activity recognition by supporting with this technique, which is our future motivation for this work.

\section{ACKNOWLEDGMENTS}

A "PSU President Scholarship" supported this work. The authors would really like to thank the officials of Prince of Songkla University, Hat Yai, 90110, Songkhla, Thailand.

\section{REFERENCES}

[1] E. Kim, S. Helal, and D. Cook, "Human Activity Recognition and Pattern Discovery," Pervasive Computing, IEEE, vol. 9, no. 1, pp. 48-53, 2010 .

[2] X. Su, H. Tong, and P. Ji, “Activity recognition with smartphone sensors," Tsinghua Science and Technology, vol. 19, no. 3, pp. 235-249, 2014.

[3] A. Avci, S. Bosch, M. Marin-Perianu, R. Marin-Perianu, and P. Havinga, "Activity Recognition Using Inertial Sensing for Healthcare, Wellbeing and Sports Applications: A Survey," Architecture of computing systems (ARCS), 201023 rd international conference, pp. 1-10, 2010.

[4] A. Bulling, U. Blanke, and B. Schiele, "A tutorial on human activity recognition using body-worn inertial sensors," ACM Computing Surveys (CSUR)., vol. 1, no. June, pp. 1-33, 2014.

[5] S. Gonz, M. Chen, S. Member, and V. C. M. Leung, "A Survey on Activity Detection and Classification Using Wearable Sensors," Technology, vol. 15, no. 4, pp. 539-549, 2017.

[6] S. Wu and Y. Song, "Human Activity Recognition on Smartphone: A Classification Analysis," , TELKOMNIKA Indonesian Journal of Electrical Engineering, vol. 12, no. 9, pp. 7041-7045, 2014.

[7] A. Alfoudery, A. A. Alkandari, and N. M. Almutairi, "Trash Basket Sensor Notification Using Arduino with Android Application," Indonesian Journal of Electrical Engineering and Computer Science(IJEECS)., vol. 10, no. 1, pp. 120-128, 2018. 
[8] Q. Zeng, B. Zhou, C. Jing, N. Kim, and Y. Kim, "A novel step counting algorithm based on acceleration and gravity sensors of a smart-phone," International Journal of Smart Home, vol. 9, no. 4, pp. 211-224, 2015.

[9] M. Derawi and P. Bours, "Gait and activity recognition using commercial phones," Computers and Security. vol. 39, no. PART B, pp. 137-144, 2013.

[10] K. Tran, T. Le, and T. Dinh, "A high-accuracy step counting algorithm for iPhones using Accelerometer," 2012 IEEE Int Symp Signal Process Inf Technol ISSPIT 2012, pp. 213-217, 2012.

[11] P. Imunisasi, D. Pada, B. Di, and W. Kerja, "Step Counting Using Smartphone Accelerometer and Fast Fourier Transform," Sigma Journal of Engineering and Natural Sciences 8 (2), 2017, 175-182, vol. 8, no. 2, pp. 1-12, 2014.

[12] R. Cuberek, W. El Ansari, K. Frmel, K. Skalik, and E. Sigmund, "A comparison of two motion sensors for the assessment of free-living physical activity of adolescents," International Journal of Environmental Research and Public Health, vol. 7, no. 4, pp. 1558-1576, 2010.

[13] H. H. Lee, S. Choi, and M. J. Lee, "Step detection robust against the dynamics of smartphones," Sensors (Switzerland), vol. 15, no. 10, pp. 27230-27250, 2015.

[14] A. K. Siddanahalli Ninge Gowda, S. R. Babu, and D. C. Sekaran, "UMOISP: Usage Mode and Orientation Invariant Smartphone Pedometer," IEEE Sensor Journal., vol. 17, no. 3, pp. 869-881, 2017.

[15] N. Ichinoseki-Sekine, Y. Kuwae, Y. Higashi, T. Fujimoto, M. Sekine, and T. Tamura, "Improving the accuracy of pedometer used by the elderly with the FFT algorithm," Medicine and Science in Sports and Exercise., vol. 38, no. 9, pp. 1674-1681, 2006.

[16] N. A. Capela, E. D. Lemaire, and N. Baddour, "Novel algorithm for a smartphone-based 6-minute walk test application: algorithm, application development, and evaluation.," Journal of neuro-engineering and rehabilitation [Internet]., vol. 12, no. 1, p. 19, 2015.

[17] C. J. Schneider, "A Natural Walking Monitor for Pulmonary Patients Using Mobile Phones," Popular Culture as Everyday Life. as Everyday Life, vol. 19, no. 4, pp. 47-56, 2016.

[18] I. Suarez, A. Jahn, C. Anderson, and K. David, "Improved Activity Recognition by Using Enriched Acceleration Data," Proceedings of the 2015 ACM International Joint Conference on Pervasive and Ubiquitous Computing (UbiComp'15), pp. 1011-1015, 2015.

[19] V. Thiyagarajan and N. P. Subramaniam, "Analysis and estimation of harmonics using wavelet technique," International Journal of Applied Engineering Research., vol. 9, no. 23, pp. 21165-21178, 2014. 